\title{
Origin and tuning of room-temperature multiferroicity in Fe-doped $\mathrm{BaTiO}_{3}$
}

\author{
Pratap Pal, ${ }^{1}$ Krishna Rudrapal, ${ }^{2}$ Sudipta Mahana, ${ }^{3}$ Satish Yadav, ${ }^{4}$ Tapas Paramanik,,${ }^{1,5}$ Shivam Mishra, ${ }^{6}$ Kiran Singh $\odot,{ }^{4,7}$ \\ Goutam Sheet, ${ }^{6}$ Dinesh Topwal, ${ }^{8}$ Ayan Roy Chaudhuri ${ }^{2,9}$ and Debraj Choudhury ${ }^{1, *}$ \\ ${ }^{1}$ Department of Physics, Indian Institute of Technology Kharagpur, West Bengal 721302, India \\ ${ }^{2}$ Advanced Technology Development Centre, Indian Institute of Technology Kharagpur, West Bengal 721302, India \\ ${ }^{3}$ Rajdhani College, Baramunda Square, Bhubaneswar 751003, India \\ ${ }^{4}$ UGC-DAE Consortium for Scientific Research, University Campus, Khandwa Road, Indore 452001, India \\ ${ }^{5}$ Department of Physics, School of Sciences, National Institute of Technology Andhra Pradesh, Tadepalligudem 534101, India \\ ${ }^{6}$ Department of Physical Sciences, Indian Institute of Science Education and Research (IISER) Mohali, \\ Sector 81, S. A. S. Nagar, Manauli, 140306, India \\ ${ }^{7}$ Department of Physics, Dr. B. R. Ambedkar National Institute of Technology, Jalandhar 144011, India \\ ${ }^{8}$ Institute of Physics, Sachivalaya Marg, Bhubaneswar 751005, India \\ ${ }^{9}$ Materials Science Centre, Indian Institute of Technology Kharagpur, West Bengal 721302, India
}

(Received 30 July 2019; revised manuscript received 25 October 2019; accepted 27 January 2020; published 11 February 2020)

\begin{abstract}
Simultaneous coexistence of room-temperature ferromagnetism and ferroelectricity in $\mathrm{Fe}$-doped $\mathrm{BaTiO}_{3}$ (BTO) is intriguing, as such Fe doping into tetragonal BTO, a room-temperature ferroelectric, results in the stabilization of its hexagonal polymorph which is ferroelectric only below $\sim 80 \mathrm{~K}$. Here, we investigate its origin and show that Fe-doped BTO has a mixed-phase room-temperature multiferroicity, where the ferromagnetism comes from the majority hexagonal phase and a minority tetragonal phase gives rise to the observed weak ferroelectricity. In order to achieve majority tetragonal phase (responsible for room-temperature ferroelectricity) in Fe-doped BTO, we investigate the role of different parameters which primarily control the paraelectric hexagonal phase stability over the ferroelectric tetragonal one and identify three major factors, namely the effect of ionic size, Jahn-Teller (JT) distortions, and oxygen-vacancies, to be primarily responsible. The effect of ionic size which can be qualitatively represented using the Goldschmidt's tolerance factor seems to be the major dictating factor for the hexagonal phase stability. The understanding of these factors not only enables us to control them but also to achieve a suitable codoped BTO compound with enhanced room-temperature multiferroic properties.
\end{abstract}

DOI: 10.1103/PhysRevB.101.064409

\section{INTRODUCTION}

The realization of room-temperature magnetoelectricmultiferroic materials which are simultaneously ferromagnetic and ferroelectric is extremely important due to their potential applicability in logic and information storage devices. However, it is extremely challenging to achieve so and there exist very few such examples [1-3]. Recently, turning $\mathrm{BaTiO}_{3}$, a room-temperature proper ferroelectric, into a potential multiferroic material has been intensively studied [4-6]. Interestingly, it has been found that multiferroicity can emerge in $\mathrm{BaTiO}_{3}$ (BTO) nanoparticles in which ferromagnetism comes from the surface states due to oxygen vacancies and the ferroelectricity from the core [4,5]. Later, it was also shown that in addition to oxygen, $\mathrm{Ti}$ or $\mathrm{Ba}$ vacancies can also induce ferromagnetism in BTO [6]. But as the induced magnetism by such vacancies is very small (few memu/gm) at room temperature [4], there were efforts towards the realization of multiferroicity in BTO by other routes like transition-metal ion $(\mathrm{Mn}, \mathrm{Fe}$, or $\mathrm{Co}$ ) doping at $\mathrm{Ti}$

*debraj@phy.iitkgp.ac.in sites [7-10]. In this regard, Fe-doped BTO, which exhibits the simultaneous coexistence of room-temperature ferromagnetism and ferroelectricity $[11,12]$, attracted a lot of attention. However, such $\mathrm{Fe}$ doping induced transformation of the ferroelectric tetragonal BTO (t-BTO) into its hexagonal polymorph (h-BTO), which is paraelectric at room temperature [13,14]. The origin of ferromagnetism, which likely arises due to the $90^{\circ}$ superexchange interaction between two $\mathrm{Fe}$ ions at Ti2 sites in the BTO hexagonal matrix [13], can be enhanced with increasing oxygen-vacancy content [15]. However, the tunability of ferromagnetism by creating oxygen vacancies can be detrimental to the insulating ferroelectric properties of BTO [16]. Thus, it is important to find other possible ways to tune the ferromagnetic properties of such an $\mathrm{Fe}$-doped BTO system. In this regard, another major issue is to recover the ferroelectric tetragonal phase from paraelectric hexagonal Fe-doped BTO, such that we can have an optimized multiferroic response from such system. Therefore, it is important to identify the key controlling parameters that facilitate the hexagonal phase formation, such that hexagonal-to-tetragonal structural phase tuning becomes possible.

The magnetic ground state of Fe-doped BTO, which is ferromagnetic at room temperature, remains contentious, as 
both paramagnetism [17] and ferromagnetism [13] have been proposed to exist at low temperature. Interestingly, a recent experimental result suggests a high-temperature ferromagnetic to low-temperature paramagnetic phase transition in Fe-doped BTO [17]. Like magnetism, the origin of roomtemperature ferroelectric polarization of Fe-doped BTO is also intriguing, since undoped h-BTO is paraelectric at roomtemperature [14]. Also, both simultaneous ferromagneticparaelectric [13] and ferromagnetic-ferroelectric [11,12] behaviors have been observed in Fe-doped BTO, thus raising the question of the origin of the observed ferroelectricity and its reproducibility. It is interesting to note that ferroelectric instability can also be triggered due to the pseudo-Jahn-Teller effect (PJTE) of the doped-Fe ${ }^{3+}\left(d^{5}\right)$ ions in the hexagonal Fedoped BTO, as recently shown in $\mathrm{LaFeO}_{3}$ [18]. Therefore, it is very critical to understand the origin of ferroelectricity and magnetic state such that the tuning of its room-temperature multiferroic properties becomes feasible.

With these goals in mind, we examine high quality polycrystalline BTO and various $\mathrm{Ba}_{1-x} A_{x} \mathrm{Ti}_{0.9} \mathrm{Fe}_{0.1} \mathrm{O}_{3}$ (where $A$ is $\mathrm{Bi}, \mathrm{Sr}$, or $\mathrm{Ca}$ and $x$ denotes the atomic concentration of doped ion at the dopant site) and $\mathrm{Ba}_{0.9} \mathrm{Bi}_{0.1} \mathrm{Ti}_{0.9} \mathrm{Mn}_{0.1} \mathrm{O}_{3}$ compounds. Here, in this paper, we show that only Fe-doped BTO is actually a mixed phase room-temperature multiferroics, where a majority hexagonal phase is ferromagnetic and a

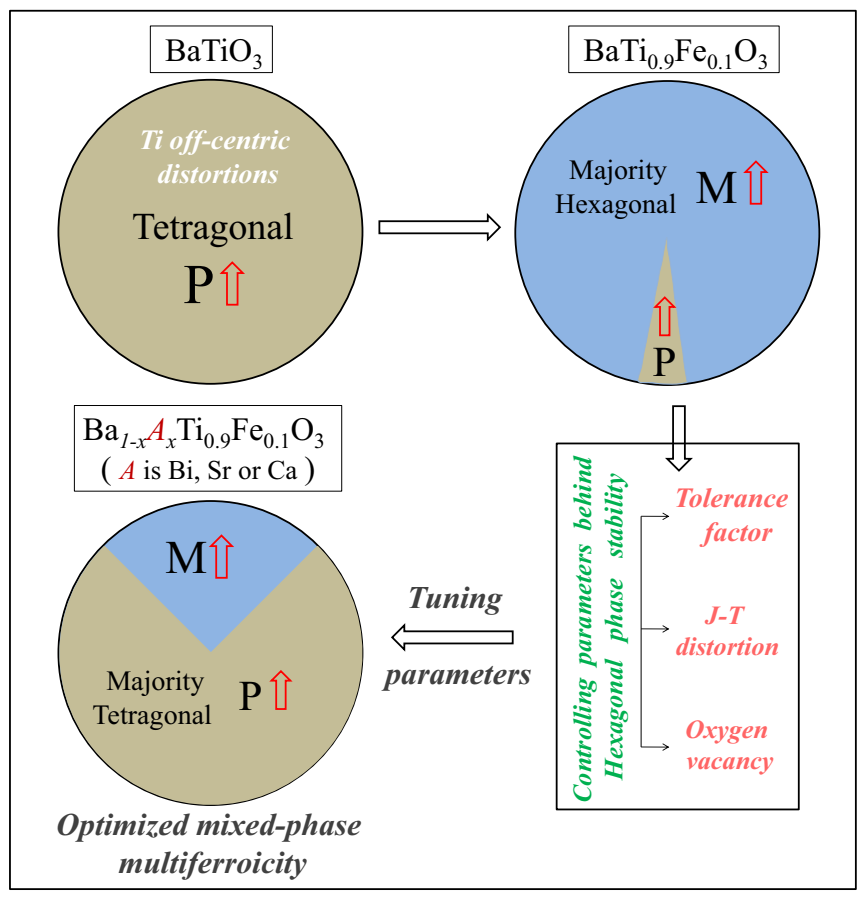

FIG. 1. Schematic representation of room-temperature mixed phase multiferroicity in Fe-doped BTO. Majority hexagonal phase, which can be controlled by tuning either Goldschmidt's tolerance factor, Jahn-Teller (JT) distortion of dopant ion, or oxygen-vacancy content, contributes to the ferromagnetism $(M \uparrow)$ of Fe-doped BTO. The observed weak ferroelectricity $(P \uparrow)$ of Fe-doped BTO arises from trace quantity of minority tetragonal phase. Identification and tuning of the control parameters which stabilize the paraelectric hexagonal phase over ferroelectric tetragonal phase in Fe-doped BTO lead to optimized mixed-phase multiferroic response from this system. minority tetragonal phase gives rise to the observed weak ferroelectricity (see Fig. 1). Subsequently, we show that along with JT distortions and oxygen vacancies, the contribution of Goldschmidt's tolerance factor on the hexagonal phase stabilization is extremely important and by manipulating these parameters, we are able to enhance the room-temperature multiferroicity.

\section{EXPERIMENTAL METHODS}

All samples are prepared in phase-pure polycrystalline form via solid-state reactions. The properly ground mixture of individual oxide elements taken in stoichiometric amounts is first annealed at $1050^{\circ} \mathrm{C}$ for $12 \mathrm{~h}$ and then at $1250^{\circ} \mathrm{C}$ for $12 \mathrm{~h}$ to get the final product. Room-temperature powder X-ray diffraction (XRD) and Raman spectroscopic measurements are carried out using $\mathrm{Cu}-K \alpha$ source and a 514-nm line of $\mathrm{Ar}^{+}$laser as an excitation source, respectively. The adopted nomenclatures for all samples are listed in Table I. The ferroelectric PUND (positive up-negative down) measurements [19] are conducted in a Radiant P-E loop tracer and the dc magnetization measurements are carried out using a Quantum Design superconducting quantum interference device magnetometer. The temperature-dependent pyroelectric current measurements are carried out using a Keithley 6517B electrometer. The sample is first cooled down to $10 \mathrm{~K}$ from room temperature under the presence of applied electric field of $400 \mathrm{~V} / \mathrm{mm}$. Then, the electric field is switched off and the electrodes are short-circuited for sufficient time to get rid of any residual surface charge effects. The sample is then heated to room temperature at a rate of $10 \mathrm{~K} / \mathrm{min}$ and we record temperature-dependent pyroelectric current. X-ray photoelectron spectroscopy (XPS) studies were carried out using an in-house PHI 5000 Versaprobe-II spectrometer and $x$-ray-absorption near-edge spectroscopy (XANES) investigations are performed at the P-65 beamline in the PETRA-III synchrotron source, DESY, Hamburg, Germany. The piezoresponse force microscopy (PFM) technique was also employed

TABLE I. All sample notations and chemical formulas along with their respective hexagonal volume phase fractions (number within brackets represents the corresponding error), as obtained from Rietveld refinement of their room-temperature powder XRD patterns. The sample notation, for example, BB05TFO $\left(\mathrm{Ba}_{0.95} \mathrm{Bi}_{0.05} \mathrm{Ti}_{0.9} \mathrm{Fe}_{0.1} \mathrm{O}_{3}\right)$, means $\mathrm{Bi}$ and $\mathrm{Fe}$ are simultaneously doped at $\mathrm{Ba}$ and $\mathrm{Ti}$ sites of BTO by 5 and 10 at. \% respectively, where the notation " 05 " denotes the at. \% of Bi doping at Ba sites.

\begin{tabular}{lcc}
\hline \hline $\begin{array}{l}\text { Sample } \\
\text { notations }\end{array}$ & $\begin{array}{c}\text { Desired chemical } \\
\text { formula }\end{array}$ & $\begin{array}{c}\text { Hexagonal phase } \\
\text { fraction }(\%)\end{array}$ \\
\hline BTFO & $\mathrm{BaTi}_{0.9} \mathrm{Fe}_{0.1} \mathrm{O}_{3}$ & $98.8(0.5)$ \\
BTFO_Q & ${\mathrm{BTFO} \mathrm{quenched} \mathrm{in} \mathrm{LN}_{2}}_{\text {BBO2TFO }}^{\mathrm{Ba}_{0.98} \mathrm{Bi}_{0.02} \mathrm{Ti}_{0.9} \mathrm{Fe}_{0.1} \mathrm{O}_{3}}$ & $99.3(0.5)$ \\
BB05TFO & $\mathrm{Ba}_{0.95} \mathrm{Bi}_{0.05} \mathrm{Ti}_{0.9} \mathrm{Fe}_{0.1} \mathrm{O}_{3}$ & $38.2(0.3)$ \\
BB10TFO & $\mathrm{Ba}_{0.9} \mathrm{Bi}_{0.1} \mathrm{Ti}_{0.9} \mathrm{Fe}_{0.1} \mathrm{O}_{3}$ & $18.6(0.2)$ \\
BS05TFO & $\mathrm{Ba}_{0.95} \mathrm{Sr}_{0.05} \mathrm{Ti}_{0.9} \mathrm{Fe}_{0.1} \mathrm{O}_{3}$ & 0 \\
BC05TFO & $\mathrm{Ba}_{0.95} \mathrm{Ca}_{0.05} \mathrm{Ti}_{0.9} \mathrm{Fe}_{0.1} \mathrm{O}_{3}$ & $24.6(0.3)$ \\
BB10TMO & $\mathrm{Ba}_{0.9} \mathrm{Bi}_{0.1} \mathrm{Ti}_{0.9} \mathrm{Mn}_{0.1} \mathrm{O}_{3}$ & $13.2(0.3)$ \\
\hline \hline
\end{tabular}


to visualize the ferroelectric domains in Fe-doped BTO, as detailed in the Supplemental Material [20].

\section{RESULTS AND DISCUSSIONS}

\section{A. Fe-doped $\mathrm{BaTiO}_{3}$ : A mixed phase multiferroics}

The room-temperature powder XRD pattern of BTFO, shown in Fig. S1 of the Supplemental Material [20], nicely matches to that of BTO hexagonal polymorph with the space group $P 6_{3} / m m c$ [21]. Though, h-BTO is paraelectric at roomtemperature [14], surprisingly, we find some finite ferroelectric polarization in hexagonal BTFO from P-E loop measurements, as shown in Fig. 2(a). However, such a cigar-shaped P-E loop which does not get saturated possibly indicates the lossy nature of the sample. However, the true ferroelectric character of BTFO could be confirmed employing room-temperature PUND (positive up and negative down) measurements (as discussed in the following), which is a well established method to extract out the intrinsic ferroelectric part (remanent contribution) from other nonferroelectric (nonremanent) contributions such as leakage current or stray capacitances [22-25]. Besides, to have direct microscopic evidence of the presence of ferroelectricity we have carried out piezoforce microscopy (PFM) as shown in Fig. S2 of the Supplemental Material [20], where we clearly see the presence of ferroelectric domains in the BTFO sample [26]. Importantly, the detailed XRD spectrum of BTFO exhibits a small but broad shoulder just after the hexagonal (110) peak, which can be accounted for by considering an additional
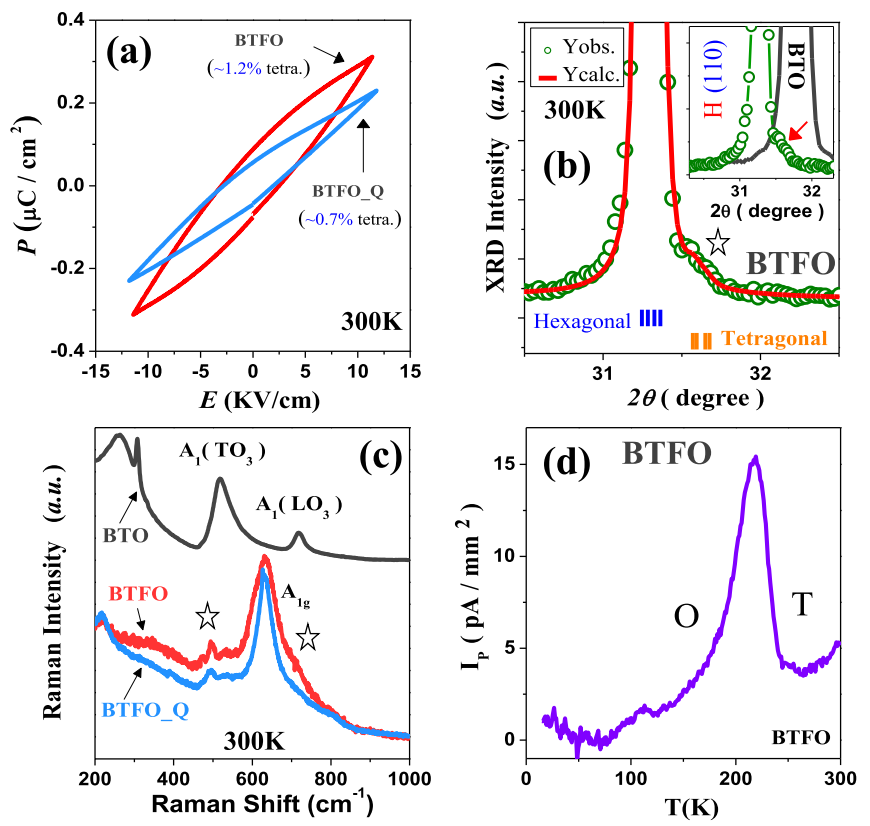

FIG. 2. (a) Comparison of room-temperature P-E loops of BTFO and BTFO_Q, (b) signature of tetragonal phase from both raw XRD pattern and Rietveld refinement, (c) Room-temperature Raman spectra of BTFO, BTFO_Q and pure BTO (star marks show the presence of tetragonal phase), and (d) Temperature-dependence of pyroelectric-current for BTFO which shows ferroelectricorthorhombic $(\mathrm{O})$ to ferroelectric-tetragonal $(\mathrm{T})$ phase transition at $218 \mathrm{~K}$ during heating.
t-BTO phase [shown in the inset to Fig. 2(b)]. The corresponding Rietveld refinement, taking mixed hexagonal and tetragonal phases, is shown in Fig. 2(b) (for full refinement see Fig. S7 [20]). Raman spectroscopy on BTFO supports this observation of a mixed phase, where the clear signature of tetragonal Raman modes $A_{1}\left(\mathrm{TO}_{3}\right)$ and $A_{1}\left(\mathrm{LO}_{3}\right)$ (shown by star marks) [27], along with the majority hexagonal $A_{1 g}$ mode [28] are observed, as shown in Fig. 2(c). These observations thus clearly indicate that BTFO is actually a mixed phase compound in which the majority is hexagonal $(\sim 98.8 \%$, obtained from Rietveld refinement) and the rest is weak tetragonal. Further, we employ pyroelectric measurement which is very sensitive to the ferroelectric phase transition [29]. Figure 2(d) clearly shows a phase transition at $\sim 218 \mathrm{~K}$ for BTFO. As BTFO has both hexagonal and tetragonal phases, with the hexagonal phase being ferroelectric only below $\sim 80 \mathrm{~K}$, such a transition in the pyrocurrent is an indication of a ferroelectric phase transition in the minority tetragonal phase from Ti off-centric displacements [30]. For such a scenario, tuning of the tetragonal phase fraction should also lead to changes in the observed room-temperature ferroelectric polarization value. BTFO_Q, which expectedly contains more oxygen vacancies than BTFO, as engineered through quenching in liquid $\mathrm{N}_{2}$ during synthesis [31] is found to contain a lower tetragonal phase fraction, as is clearly seen through the weaker tetragonal Raman peaks in Fig. 2(c). Following the above conjecture, BTFO_Q indeed possesses a lower roomtemperature polarization value when compared to BTFO, as shown in Fig. 2(a). This is further highlighted using a comparison of the true remanent ferroelectric polarization values between BTFO and BTFO_Q, as extracted using the PUND technique. Figure 3(a) displays room-temperature PUND polarization data of BTFO, where the preset, switching, and nonswitching applied voltage pulses are schematically shown (see also Fig. S3 for a detailed PUND mechanism [20]). The negative pulse- 1 is necessary to preset the material. The application of the positive switching pulse 2 thereby induces switching of both the ferroelectric component along with switching of the linear nonferroelectric (nonremanent) contributions. As the ferroelectric dipoles are already aligned and do not relax during the intermittent delay time, the following positive pulse 3 cannot induce any further switching of the ferroelectric component and only induces further polarization of the nonferroelectric (nonremanent) components. Thus, the intrinsic ferroelectric remanent polarization $(d P r)$ can be readily obtained by subtracting the polarization $\left(P^{\wedge} r\right)$ value, obtained after application of pulse 3 , from the polarization value $\left(P^{\star} r\right)$, obtained after the application of pulse 2. A comparison of the $d P r$ values for BTFO and BTFO_Q, obtained using the above method, are shown in Fig. 3(b). We indeed see the presence and switching of remanent polarization $(d P r)$ for both BTFO and BTFO_Q as shown in Fig. 3(b) (for detailed discussions of ferroelectric PUND data of these two compounds see Fig. S4 [20]). The PUND data clearly show a smaller remanent polarization value for BTFO_Q in comparison to BTFO [a similar trend as observed in Fig. 2(a)], which can be expected only if the ferroelectric polarization originates from the tetragonal phase. Now, to understand the presence of oxygen vacancies, we have carried out room-temperature XPS measurements. It is well known that the higher binding-energy shoulder of 

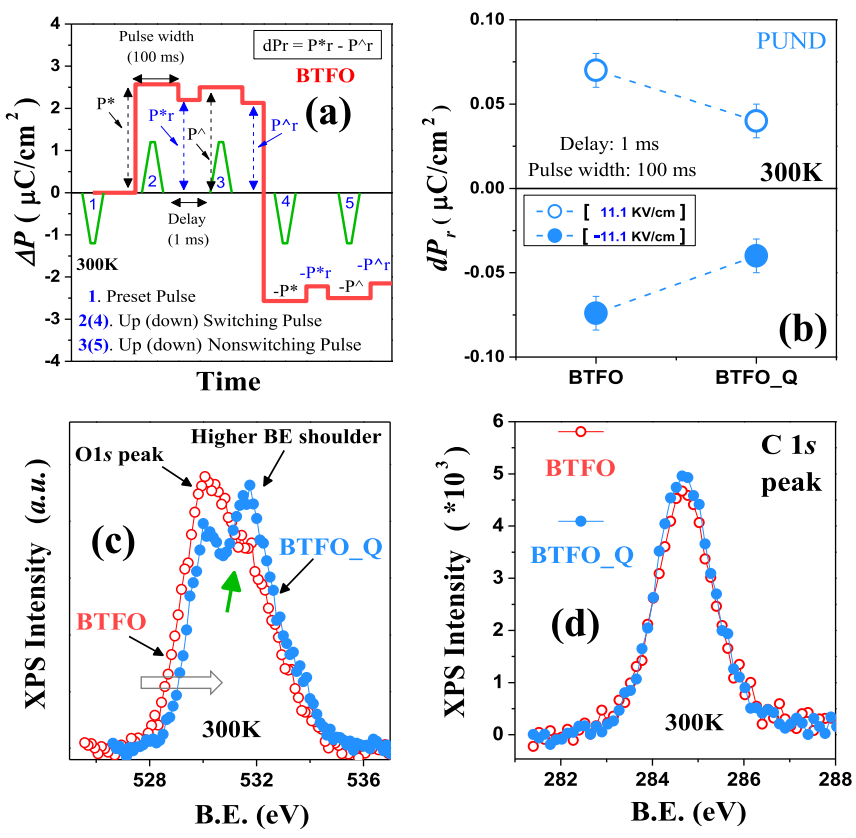

FIG. 3. (a) Room-temperature PUND data (red line) of BTFO, where the applied voltage pulses (green ones) are schematically shown, (b) variation and switching of the room-temperature remanent polarization of BTFO and BTFO_Q as obtained from PUND measurement; (c) shows corresponding room-temperature O-1s XPS spectra and (d) shows their room-temperature C-1s XPS spectra.

O- $1 s$ peak arises from defect sites, like oxygen vacancies and adsorbed hydrocarbons [32]. The presence of identical weightages of C- $1 s$ spectra for both BTFO and BTFO_Q, as seen in Fig. 3(d), shows the presence of similar amounts of adsorbed hydrocarbons on the surface of these samples. However, the higher binding-energy shoulder of O- $1 s$ peak for BTFO_Q has significantly larger intensity as compared to BTFO, as shown in Fig. 3(c), thereby, clearly bringing out the presence of more oxygen vacancies in the BTFO_Q sample $[32,33]$. This is also supported by the movement of the O-1s peak to higher binding energy in BTFO_Q as compared to BTFO [see Fig. 3(c)] [34]. Interestingly, while stoichiometric BTFO should contain only nominal $\mathrm{Fe}^{4+}$ (the ground state in this case is primarily a mixture of $d^{4}$ and $d^{5} \underline{L}$ states with $\underline{L}$ representing an $\mathrm{O} 2 p$ hole [35]) ions, the presence of oxygen vacancies is correlated with the conversion of some nominal $\mathrm{Fe}^{4+}$ ions into $\mathrm{Fe}^{3+}$ state due to charge neutrality $[21,36]$. Thus, the presence of a smaller room-temperature ferroelectric polarization value in BTFO_Q, which contains more $\mathrm{Fe}^{3+}$ ion content than BTFO, also rules out PJTE as the mechanism for the observed ferroelectric polarization of BTFO.

\section{B. Coexistence of ferromagnetism and paramagnetism in Fe-doped $\mathrm{BaTiO}_{3}$}

To understand the magnetic ground state, we have performed isothermal $M-H$ measurements from room temperature down to $10 \mathrm{~K}$, as shown in Fig. 4(a). At room temperature, there is a clear ferromagnetic $M-H$ loop for BTFO, which is understood to arise from ferromagnetic exchange interactions
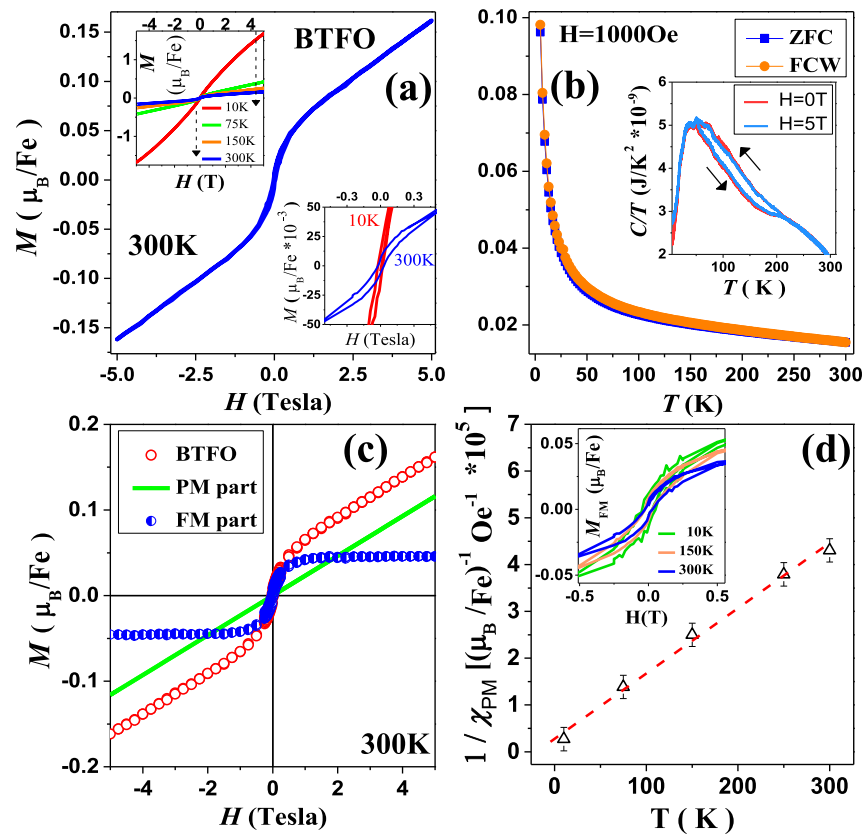

FIG. 4. (a) Isothermal $M-H$ loops of BTFO and (b) variation of magnetization $(M)$ with temperature $(T)$. Inset to (b) shows the heat-capacity vs $T$ plot, both in absence and presence of magnetic field of 5 T. (c) Decomposition of room-temperature $M-H$ plot into paramagnetic and ferromagnetic parts and (d) displays the linear behavior of the paramagnetic inverse susceptibility with temperature. Inset to (d) shows the existence of ferromagnetic loops at all temperatures.

in the hexagonal phase [13,37]. At $10 \mathrm{~K}$, the $M-H$ loop, however, looks like a Brillouin function, as expected for paramagnetic order, as shown in Fig. 4(a). Such a behavior has previously been attributed to a room-temperature ferromagnetic to low-temperature paramagnetic phase transition for BTFO [17], which is quite unusual, and, thus, we investigated it further. On carefully analyzing the $10-\mathrm{K} M-H$ loop near the low-field region, we do find the existence of a clear hysteresis loop, also suggesting the presence of ferromagnetic order at low temperature. Also, although a clear $M-H$ hysteresis loop is observed at $300 \mathrm{~K}$, unlike a ferromagnet, it does not saturate and continues to rise linearly in the high-field region. Further, no clear ferromagnetic to low-temperature paramagnetic phase transition is observed in the $M$ vs $T$ plot, as shown in Fig. 4(b) or even in the $d M / d T$ vs $T$ plot. The absence of any magnetic transition is also supported by the heat-capacity data, which do not show any significant changes in the presence of magnetic field $(H=5 \mathrm{~T})$ [as shown in the inset to Fig. 4(b)], thereby suggesting that the transitions observed in the intermediate temperature window are of structural origin. The structural origin of the heat-capacity anomalies are also evident from the temperature-dependent dielectric constant data as shown in Fig. S5 of the Supplemental Material [20] (see also Refs. [13,38] therein). A structural transition can indeed be expected from the high-temperature hexagonal to low-temperature orthorhombic phase around similar temperature [13]. It is also important to note that often such dilute magnetic semiconductor (DMS) systems are associated with both paramagnetic and ferromagnetic signals 
$[13,39]$, as seems to be the case for BTFO. We thus fit the high-field magnetization data at $300 \mathrm{~K}$ with a linear equation and the obtained slope gives us the estimate of the paramagnetic contribution $(M=\chi H)$. On performing similar analyses at all temperatures, we find the expected linear $(1 / \chi-T)$ dependence of the extracted paramagnetic contributions, as shown in Fig. 4(d). Simultaneously, the presence of clear $M-H$ loops [obtained by subtracting the paramagnetic linear contribution, as shown by an illustrative example in Fig. 4(c)] at all temperatures from $300 \mathrm{~K}$ down to $10 \mathrm{~K}$ suggest that ferromagnetic order exists down to the lowest temperature. Thus, our data clearly suggest coexistence of both ferromagnetic and paramagnetic regions in BTFO at all temperatures.

\section{Role of controlling parameters behind hexagonal phase stability in $\mathrm{Fe}$-doped $\mathrm{BaTiO}_{3}$}

We have thus elucidated that mixed phase multiferroicity exists in Fe-doped BTO, with weak ferroelectricity arising from the minority tetragonal phase. To enhance ferroelectricity in doped BTO, it is important to investigate the role of different parameters that play a crucial part in the hexagonal phase stability in BTFO. For this purpose, we examine the BTFO, BTFO_Q, BB02TFO, BB05TFO, BS05TFO, BC05TFO, BB10TFO, and BB10TMO samples. Room-temperature XRD spectra are shown in Fig. 5(a) (for full spectra see Fig. S6 [20]), where we can clearly see variations of hexagonal first-order peak intensity for different
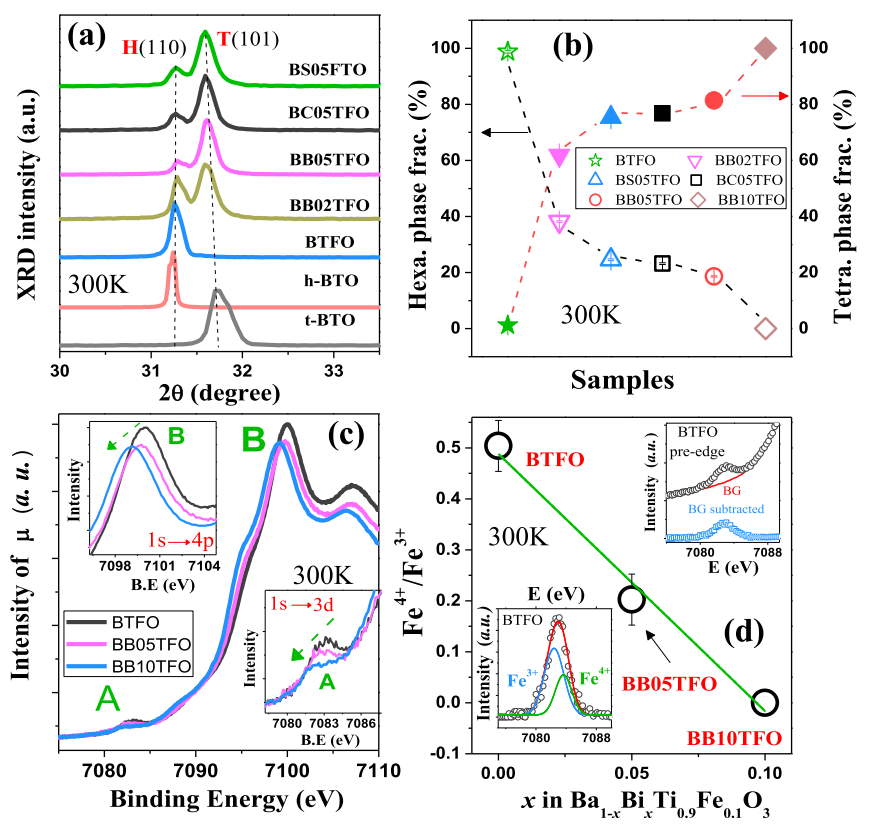

FIG. 5. (a) Room-temperature XRD spectra (only h-BTO reference XRD is taken from ICSD database) of the samples. (b) Variation of hexagonal phase (left $y$ axis) and tetragonal phase (right $y$ axis) fractions $(\%)$ for different sample [(Hexa. + Tetra) phase fractions = $100 \%$ ]. (c) Room-temperature Fe $K$-edge XANES spectra of BTFO, BB05TFO, and BB10TFO. The top and bottom insets show the evolution of main edge and pre-edge regions with doping. (d) Quantification of nominal $\mathrm{Fe}^{4+}$ to $\mathrm{Fe}^{3+}$ ratio, through background (BG) subtraction (top inset) and deconvolution of the pre-edge region into $\mathrm{Fe}^{3+}$ and nominal $\mathrm{Fe}^{4+}$ contributions (bottom inset). samples. The percentage of hexagonal and tetragonal phases present in these samples are determined from Rietveld refinement [40-43] using the FULLPROF suite (for refinement details see Secs. VII-IX of the Supplemental Material [20]) and are tabulated in Table I and also shown in Fig. 5(b). The hexagonality which is here found to be maximum for only Fe-doped BTO (BTFO), gets further enhanced in BTFO_Q due to the presence of more oxygen vacancies compared to BTFO. Such strong hexagonal phase stabilization over tetragonal BTO on Fe doping is known to be primarily dictated by Jahn-Teller distortion of nominally $\mathrm{Fe}^{4+}$ ions and a further secondary contribution comes from oxygen vacancies $[21,44,45]$. As $\mathrm{Ba}$ is gradually replaced by $\mathrm{Bi}, \mathrm{Sr}$, or $\mathrm{Ca}$, the hexagonal phase fraction is found to get strongly suppressed along with a concomitant tetragonal BTO phase recovery, as shown in Fig. 5(b) (see also Table I). To understand the role of different controlling parameters on the hexagonal phase stability, we first consider the three compounds BB05TFO ( $\mathrm{Bi}$ doped at the $\mathrm{Ba}$ site), BS05TFO ( $\mathrm{Sr}$ doped at the $\mathrm{Ba}$ site), and $\mathrm{BC} 05 \mathrm{TFO}$ ( $\mathrm{Ca}$ doped at the $\mathrm{Ba}$ site). The reduction of hexagonal phase fraction in BB05TFO can be understood using the framework of previous studies, as stated earlier, since $\mathrm{Bi}^{3+}$ substitution in the place of $\mathrm{Ba}^{2+}$ reduces oxygen vacancies as well as JT active nominally $\mathrm{Fe}^{4+}$ content to $\mathrm{Fe}^{3+}$ $\left(3 d^{5}\right)$ to maintain charge neutrality. Such observations are further validated from the systematic trend of hexagonal phase tuning from $\sim 98.8 \%$ in the case of BTFO (where only Fe is doped) to $\sim 0 \%$ for BB10TFO (where $\mathrm{Bi}$ and $\mathrm{Fe}$ are doped in equal amounts) through $\sim 38.2 \%$ in BB02TFO and $\sim 18.6 \%$ in BB05TFO [see Fig. 5(b) and Table I]. However, the sharp reduction of the hexagonal phase by significant amounts on $\mathrm{Sr}^{2+}$ or $\mathrm{Ca}^{2+}$ substitutions in BS05TFO and BC05TFO is quite surprising and cannot be understood using the earlier framework, as doping with $\mathrm{Sr}^{2+}$ and $\mathrm{Ca}^{2+}$, being isovalent to $\mathrm{Ba}^{2+}$ (only difference is their ionic sizes), is neither expected to vary the oxygen vacancies nor the JT active ion content. Thus, a holistic understanding of the role of ionic size effects, quantified through the Goldschmidt tolerance factor, which provides an effective way of investigating phase stabilities of perovskite oxides [46], along with oxygen vacancies and JT distortions to the hexagonal phase stabilization, becomes crucial.

Since the ionic sizes depend on the ion valency, we have carried out XANES measurements to investigate the Fe-ion valency. Ti was found to remain in the $4+$ state in all these samples, as seen by our XPS studies (see Fig. S8 [20]; also, note Ref. [47] therein). The normalized Fe $K$-edge XANES spectra of BTFO, BB05TFO, and BB10TFO are shown in Fig. 5(c). We can subdivide the spectra into two regions: The pre-edge region $(A: 1 s \rightarrow 3 d$ excitation) and the main edge region ( $B: 1 s \rightarrow 4 p$ excitation) [48]. Here, features $A$ and $B$ are observed to shift towards lower energy with increasing $\mathrm{Bi}(+3)$ doping, which is expected if the oxidation state of $\mathrm{Fe}$ steadily decreases from nominal $\mathrm{Fe}^{4+}$ to $\mathrm{Fe}^{3+}$ [49]. For BB10TFO with equal amounts of $\mathrm{Bi}^{3+}$ and $\mathrm{Fe}$ doping, $\mathrm{Fe}$ is expected to be in the $\mathrm{Fe}^{3+}$ state. Hence, these XANES observations indicate the presence of nominal $\mathrm{Fe}^{4+}$ to some extent in BTFO, which gets decreased in amount with $\mathrm{Bi}$ doping. To quantify a nominal $\mathrm{Fe}^{4+}$ to $\mathrm{Fe}^{3+}$ ratio, we investigate the pre-edge peak region [49]. The tentative 

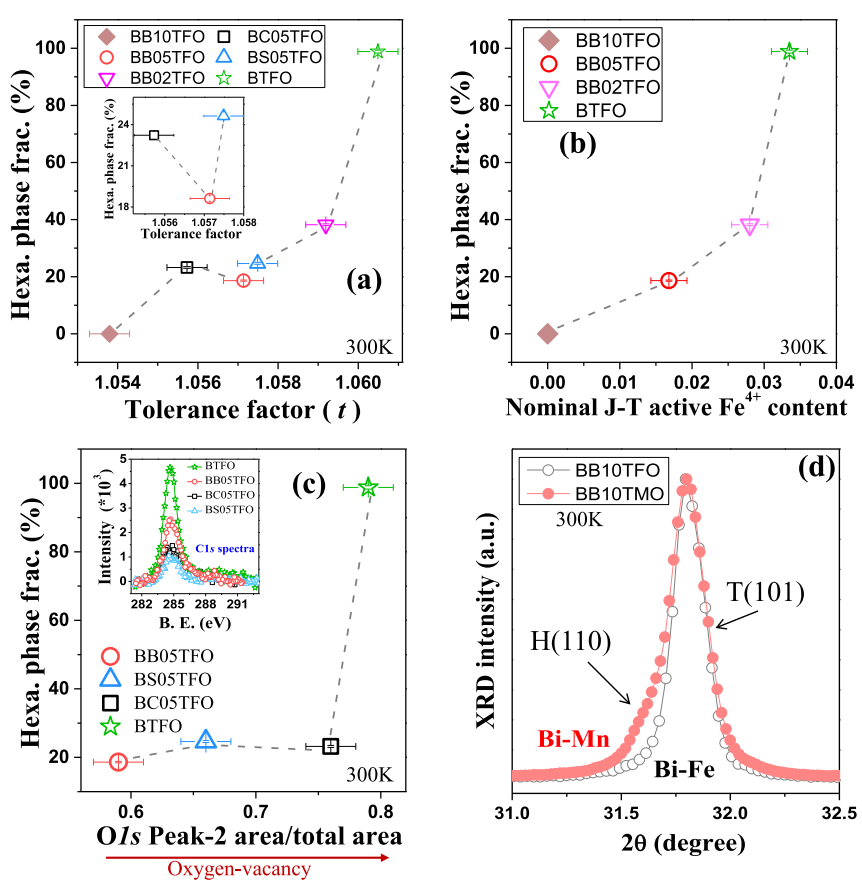

(e)

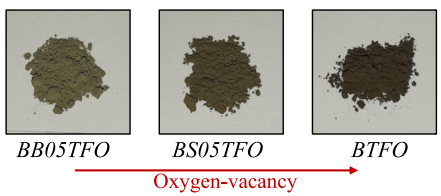

FIG. 6. Variation of hexagonal phase fractions (\%) [100, tetragonal phase fraction $(\%)$ ] of the samples with their corresponding (a) Goldschmidt's tolerance factor, (b) the nominal JT active $\mathrm{Fe}^{4+}$ content present, (c) O-1s higher binding-energy peak (peak 2) area divided by the total area, which corresponds to the oxygen vacancy in the system. The inset to (c) displays their C- $1 s$ spectra. (d) Roomtemperature XRD spectra of BB10TFO and BB10TMO, where clear presence of significant amount of hexagonal phase in BB10TMO indicates the role of JT distortion due to $\mathrm{Mn}^{3+}\left(3 d^{4}\right)$ towards hexagonal phase stabilization and (e) shows variation of sample color, dictating the change in oxygen vacancy.

amounts of nominal $\mathrm{Fe}^{4+}$ in these samples, as calculated from background subtraction and subsequent deconvolution of the pre-edge peak into $\mathrm{Fe}^{3+}$ and nominal $\mathrm{Fe}^{4+}$ contributions, are shown in Fig. 5(d). From this calibration, now, we calculate the Goldschmidt tolerance factor for all samples.

Figure 6(a) shows the variation of hexagonal phase fraction with the corresponding tolerance factor for various samples: The correspondence seems near direct; an increase in the tolerance factor generally leads to an increase in the hexagonal phase fraction. It is interesting to note that hexagonality, which gets suppressed in BS05TFO $\left(\mathrm{Ba}^{2+}\right.$ is partially replaced by $\mathrm{Sr}^{2+}$ ) compared to BTFO (Ba is not replaced), gets further suppressed in BC05TFO where $\mathrm{Ba}^{2+}$ is replaced by $\mathrm{Ca}^{2+}$ (having smaller ionic radii than $\mathrm{Sr}^{2+}$ ) due to a smaller tolerance factor of BC05TFO as compared to BS05TFO [as shown in the inset to Fig. 6(a)]. Such observations strongly indicate that the Goldschmidt tolerance factor or the ionic size effect play a crucial role behind the hexagonal phase stability. Against the above general trend, BB05TFO has smaller hexagonal phase fraction when compared to BC05TFO, though the tolerance factor of the former is more than that of the latter [as shown in the inset to Fig. 6(a)]. To understand this, we need to also consider the effect of the other controlling parameters like oxygen vacancies or JT distortions, associated with nominal $\mathrm{Fe}^{4+}$ ions, on the hexagonal phase stability.

In Fig. 6(b) we note that as JT active nominal $\mathrm{Fe}^{4+}$ content (as obtained from room-temperature $\mathrm{Fe}$ $K$ edge XANES measurements) increases, the hexagonal phase gets more stabilized. To elucidate it further, we have compared room-temperature XRD spectra of BB10TFO $\left(\mathrm{Ba}_{0.9} \mathrm{Bi}_{0.1} \mathrm{Ti}_{0.9} \mathrm{Fe}_{0.1} \mathrm{O}_{3}\right)$ with BB10TMO $\left(\mathrm{Ba}_{0.9} \mathrm{Bi}_{0.1} \mathrm{Ti}_{0.9} \mathrm{Mn}_{0.1} \mathrm{O}_{3}\right)$ in Fig. 6(d), where the former (BB10TFO) contains only the Jahn-Teller inactive $\mathrm{Fe}^{3+}\left(3 d^{5}\right)$ ion, and the latter (BB10TMO) contains the Jahn-Teller active $\mathrm{Mn}^{3+}\left(3 d^{4}\right)$ ion. In agreement with the trend of increasing hexagonality with increasing content of the Jahn-Teller active ion, it is found that while BB10TFO is completely tetragonal, BB10TMO has a significant amount of hexagonal phase, though both of them have an identical tolerance factor $\left(\mathrm{Fe}^{3+}\right.$ and $\mathrm{Mn}^{3+}$ have the same ionic radii).

Next, to understand the role of oxygen vacancies towards hexagonal phase stability, we have carried out roomtemperature XPS studies on O- $1 s$ spectra (see Fig. S9 of [20]). As discussed earlier, the higher binding-energy shoulder (peak 2) of O-1s spectra corresponds to oxygen vacancies and adsorbed hydrocarbons [32]. Upon fitting the O-1s spectra with two peaks, we plot the change in the hexagonal phase with the corresponding O- $1 s$ peak- 2 area (normalized by the total area under the O- $1 s$ peak region after Shirley background subtraction), as shown in Fig. 6(c) (the inset shows the corresponding $\mathrm{C}-1 s$ spectra which measures the relative adsorbed hydrocarbon content). The results show that there is a strong reduction of oxygen vacancies on $\mathrm{Bi}^{3+}$ doping for $\mathrm{Ba}^{2+}$, which can be expected since increased $\mathrm{Bi}^{3+}$ ion content naturally promotes the more stable $\mathrm{Fe}^{3+}$ ion content without the need for any oxygen vacancies. Surprisingly, we also notice a reduction of oxygen vacancies in isovalent substituted BC05TFO and BS05TFO compounds. This observation can only be understood by considering the suppression of the hexagonal phase in the $\mathrm{Sr}$ - and $\mathrm{Ca}$-doped samples as it has been argued that oxygen vacancies tend to be hosted in the hexagonal phase [50,51] rather than the tetragonal phase. Further, such reduction of oxygen-vacancy content in the samples where the $A$ site $\mathrm{Ba}$ ion is replaced by other ions ( $\mathrm{Bi}, \mathrm{Sr}$, or $\mathrm{Ca}$ ) can also be visualized from the sample color change as shown in Fig. 6(e), where it seems that the darker the sample is, the more is the oxygen-vacancy content. A comparison between Figs. 6(a) and 6(c) shows that though $\mathrm{BC} 05 \mathrm{TFO}$ has more oxygen vacancies than BS05TFO, it has a lesser hexagonal phase fraction, which seems primarily due to the smaller tolerance factor of the former compared to the latter. Similarly, since BB05TFO has a lower oxygen vacancy and Jahn-Teller active ion content compared to BC05TFO, the former has a slightly smaller hexagonal phase fraction compared to the latter, as seen in the inset to Fig. 6(a).

Therefore, from these discussions it is clear that along with oxygen vacancy and JT distortions, there is a significant role of ionic size towards hexagonal phase stabilization in such transition-metal-doped $\mathrm{BaTiO}_{3}$. It is also extremely important 
to understand which of these controlling parameters plays a more dominant role to destabilize the hexagonal phase, because then by controllably manipulating that parameter we can more effectively control the hexagonal to tetragonal structural phase ratio, which is critical for achieving better optimized room-temperature multiferroicity in such an Fe-doped BTO system. To highlight this, we consider two compounds, BTFO and BB05TFO, for which we see that the hexagonal phase fraction is reduced by $\sim 80 \%$ in the latter compound compared to the former due to only a $\sim 0.3 \%$ change in tolerance factor, whereas the respective change in other controlling parameters (namely oxygen vacancy content and Jahn-Teller active ion content) is quite large. Such an observation clearly indicates that the Goldschmidt tolerance factor plays the dictating role behind the hexagonal phase stability.

\section{Tuning of room-temperature multiferroicity of Fe-doped $\mathrm{BaTiO}_{3}$}

For the study of multiferroic properties, we have carried out room-temperature $M-H$ measurements on BTFO, BB02TFO, BS05TFO, and BB10TFO. The saturated magnetization $\left(M_{S}\right)$ of the extracted ferromagnetic part for these samples along with the corresponding hexagonal phase fraction $(\%)$ present in the samples are shown in Fig. 7(a). Here,
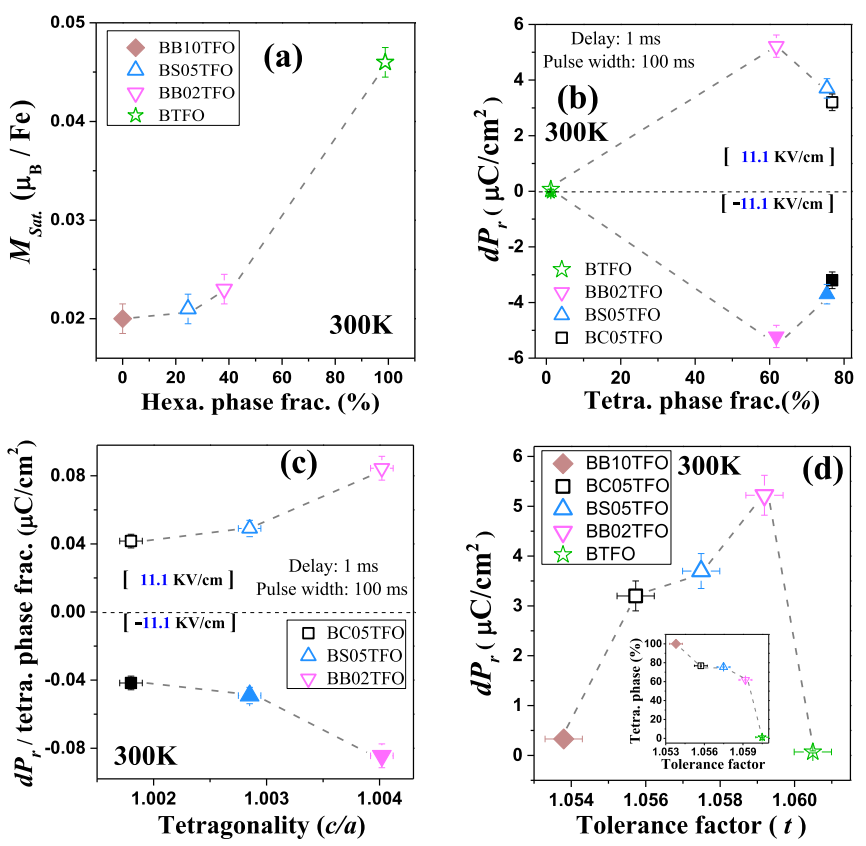

FIG. 7. (a) Variation of room-temperature saturation magnetization $\left(M_{S}\right)$ of the extracted ferromagnetic part with the corresponding sample hexagonal phase fraction; (b) variation as well as switching of the room-temperature remanent polarization $(d P r)$ as obtained from PUND measurements with the corresponding tetragonal phase fraction (\%); (c) variation of remanent polarization per unit tetragonal phase fraction $(\%)$ with the corresponding sample tetragonality $(c / a)$; (d) change in remanent polarization for samples with their corresponding Goldschmidt's tolerance factor, where its inset shows how tetragonal phase fraction $(\%)$ varies with their respective tolerance factor.
TABLE II. All samples with their corresponding tetragonal volume phase fractions, room-temperature tetragonality $(c / a)$ as obtained from Rietveld refinement, and room temperature intrinsic remanent ferroelectric polarization as observed in PUND measurements. Number within brackets in the second column represents the error in the corresponding tetragonal phase percentage.

\begin{tabular}{lccc}
\hline \hline $\begin{array}{l}\text { Sample } \\
\text { notations }\end{array}$ & $\begin{array}{c}\text { Tetragonal } \\
\text { phase fraction } \\
(\%)\end{array}$ & $\begin{array}{c}\text { Tetragonality } \\
(c / a)\end{array}$ & $\begin{array}{c}\text { Remanent } \\
\text { polarization } \\
d P r\left(\mu \mathrm{C} / \mathrm{cm}^{2}\right)\end{array}$ \\
\hline BTFO & $1.2(0.1)$ & $1.0018(1)$ & $0.07 \pm 0.01$ \\
BTFO_Q & $0.7(0.2)$ & $1.0016(1)$ & $0.04 \pm 0.01$ \\
BB02TFO & $61.8(0.4)$ & $1.0040(1)$ & $5.2 \pm 0.4$ \\
BB05TFO & $81.4(0.3)$ & $1.0037(1)$ & $4.3 \pm 0.3$ \\
BB10TFO & $100(0.5)$ & $1.0002(1)$ & $0.33 \pm 0.03$ \\
BS05TFO & $75.4(0.5)$ & $1.0029(1)$ & $3.7 \pm 0.4$ \\
BC05TFO & $76.8(0.6)$ & $1.0018(1)$ & $3.2 \pm 0.3$ \\
\hline \hline
\end{tabular}

the change in $M_{S}$ closely reflects the change in the respective hexagonal volume phase fraction. It is already known that the ferromagnetism in Fe-doped BTO arises due to $90^{\circ}$ superexchange between two $\mathrm{Fe} 2$ ions at Ti2 sites in the hexagonal unit [13], hence the tuning of such hexagonal phase also leads to the tuning of corresponding ferromagnetic properties, as seen in Fig. 7(a).

Subsequently, we have studied the intrinsic ferroelectric properties of these samples employing the PUND technique. Figure 7(b) shows the variation as well as ferroelectric switching of remanent polarization $(d P r)$ with the corresponding sample tetragonal phase fraction $(\%)$. Here, we see that BB02TFO has a maximum polarization $\left(\sim 5.2 \mu \mathrm{C} / \mathrm{cm}^{2}\right)$ which is $\sim 74$ times more than that of BTFO, which is quite remarkable, as the recovery of ferroelectricity in such an Fe-doped BTO system by such a margin can be extremely helpful in the engineering of room-temperature multiferroicity. Surprisingly, though BC05TFO has more tetragonal phase fractions than BS05TFO and BB02TFO, it shows lesser ferroelectric polarization, as seen in Fig. 7(b). To understand this, we have plotted ferroelectric polarization normalized to the tetragonal phase percentage present in the sample along with the corresponding tetragonality $(c / a)$ as shown in Fig. 7(c), where we find that the order of sample tetragonality is BB02TFO > BS05TFO > BC05TFO, which clearly reflects the origin of smaller polarization in BC05TFO. Thus, the sample tetragonality, evidently, seems to play a dominant role in the ferroelectric properties (see also Table II). The strong dependency of the recovered ferroelectricity of all these compounds on the tetragonal phase fraction and tetragonality $(c / a)$ indicates that the ferroelectricity originates from the $\mathrm{Ti}$ off-centric distortions [30]. We further note that all the investigated compounds have the Goldschmidt tolerance factor $\geqslant 1.054$, which is larger than the limit necessary to cause octahedral tilting [52,53], as observed in orthorhombic $\mathrm{CaMnO}_{3}$ [54], thus any role of such octahedral tilting in the observed ferroelectricity in our samples can be ruled out.

It is interesting to note that there is a range of Goldschmidt tolerance factor for which the sample shows optimized ferroelectric polarization, as seen in Fig. 7(d). Such an optimized range of tolerance factor for the best ferroelectric Fe-doped 
BTO compound arises because for a higher tolerance factor the sample tends to have more hexagonality, whereas for smaller tolerance factors, the sample goes to the cubic limit. Thus, the understanding of the origin of ferroelectricity and the controlling parameters for the hexagonal phase stability in Fe-doped BTO enables us to controllably tune the roomtemperature mixed phase multiferroicity, where magnetism is attributed to the hexagonal phase and the ferroelectricity to the tetragonal phase.

\section{CONCLUSIONS}

In summary, we have investigated the room-temperature multiferroicity in Fe-doped BTO and have shown that it is of mixed phase origin, where the ferromagnetism is associated with the majority hexagonal phase and ferroelectricity to the minority tetragonal phase. We have also elucidated that ferromagnetic and paramagnetic phases coexist at all temperatures in BTFO. By examining various codoped BTO compounds, we show that compared to JT distortions and oxygen vacancies, the Goldschmidt tolerance factor or the ionic size effect has a more decisive role on the stabilization of paraelectric hexagonal phase over the ferroelectric tetragonal one. By controlling these parameters, we successfully achieve tuning of the room-temperature mixed phase multiferroicity in engineered codoped BTO compounds.

\section{ACKNOWLEDGMENTS}

We acknowledge the use of XPS under the DST-FIST facility in the Department of Physics, IIT Kharagpur for this work. P.P. would like to acknowledge the financial support from MHRD, India. D.C. would like to acknowledge SERB, DST, India (funding under project file no. ECR/2016/000019) and BRNS, DAE [funding through sanction number 37(3)/20/23/2016-BRNS] for financial support. D.T. would like to gratefully acknowledge financial support by DST provided with in the framework of the India@DESY collaboration.
[1] J. F. Scott, NPG Asia Mater. 5, e72 (2013).

[2] T. Katayama, S. Yasui, Y. Hamasaki, T. Shiraishi, A. Akama, T. Kiguchi, and M. Itoch, Adv. Funct. Mater. 28, 1704789 (2017).

[3] N. A. Hill, J. Phys. Chem. B 104, 6694 (2000).

[4] R. V. K. Mangalam, N. Ray, U. V. Waghmare, A. Sundaresan, and C. N. R. Rao, Solid State Commun. 149, 1 (2009).

[5] S. Ramakanth and K. C. J. Raju, Solid State Commun. 187, 59 (2014).

[6] A. Raeliarijaona and H. Fu, Phys. Rev. B 96, 144431 (2017).

[7] Y. H. Lin, J. Yuan, S. Zhang, Y. Zhang, J. Liu, Y. Wang, and C. W. Nan, Appl. Phys. Lett. 95, 033105 (2009).

[8] R. Maier, J. L. Cohn, J. J. Neumeier, and L. A. Bendersky, Appl. Phys. Lett. 78, 2536 (2001).

[9] H. K. Chandra, K. Gupta, A. K. Nandy, and P. Mahadevan, Phys. Rev. B 87, 214110 (2013).

[10] L. B. Luo, Y. G. Zhao, H. F. Tian, J. J. Yang, J. Q. Li, J. J. Ding, B. He, S. Q. Wei, and C. Gao, Phys. Rev. B 79, 115210 (2009).

[11] B. Xu, K. B. Yin, J. Lin, Y. D. Xia, X. G. Wan, J. Yin, X. J. Bai, J. Du, and Z. G. Liu, Phys. Rev. B 79, 134109 (2009).

[12] A. Rani, J. Kolte, S. S. Vadla, and P. Gopalan, Ceram. Int. 42, 8010 (2016).

[13] S. Ray, P. Mahadevan, S. Mandal, S. R. Krishnakumar, C. S. Kuroda, T. Sasaki, T. Taniyama, and M. Itoh, Phys. Rev. B 77, 104416 (2008).

[14] E. Sawaguchi, Y. Akishige, and M. Kobayashi, Jpn. J. Appl. Phys. 24, 252 (1985).

[15] T. Chakraborty, S. Ray, and M. Itoh, Phys. Rev. B 83, 144407 (2011)

[16] A. Sagdeo, A. Nagwanshi, P. Pokhriyal, A. K. Sinha, P. Rajput, V. Mishra, and P. R. Sagdeo, J. Appl. Phys. 123, 161424 (2018).

[17] X. K. Wei, T. Zou, F. Wang, Q. H. Zhang, Y. Sun, L. Gu, A. Hirata, M. W. Chen, Y. Yao, C. Q. Jin, and R. C. Yu, J. Appl. Phys. 111, 073904 (2012).

[18] L. Weston, X. Y. Cui, S. P. Ringer, and C. Stampfl, Phys. Rev. B 93, 165210 (2016).
[19] J. T. Evans, Characterizing Ferroelectric Materials (Radiant Technology Inc., New Mexico, 2011).

[20] See Supplemental Material at http://link.aps.org/supplemental/ 10.1103/PhysRevB.101.064409 for PFM data, details of PUND mechanism along with the PUND data, structural and refinement details, dielectric data, and XPS analyses.

[21] R. Böttcher, H. T. Langhammer, T. Müller, and H. P. Abicht, J. Phys.: Condens. Matter 20, 505209 (2008).

[22] J. F. Scott, C. A. Araujo, H. B. Meadows, L. D. McMillan, and A. Shawabkeh, J. Appl. Phys. 66, 1444 (1989).

[23] Y. S. Chai, Y. S. Oh, L. J. Wang, N. Manivannan, S. M. Feng, Y. S. Yang, L. Q. Yan, C. Q. Jin, and K. H. Kim, Phys. Rev. B 85, 184406 (2012).

[24] B. Rajeswaran, D. I. Khomskii, A. K. Zvezdin, C. N. R. Rao, and A. Sundaresan, Phys. Rev. B 86, 214409 (2012).

[25] S. M. Feng, Y. S. Chai, J. L. Zhu, N. Manivannan, Y. S. Oh, L. J. Wang, Y. S. Yang, C. Q. Jin, and K. H. Kim, New J. Phys. 12, 073006 (2010).

[26] D. Liu, C. Tian, C. Ma, L. Luo, Y. Tang, T. Wang, W. Shi, D. Sun, and F. Wang, Scr. Mater. 123, 64 (2016).

[27] C. H. Perry and D. B. Hall, Phys. Rev. Lett. 15, 700 (1965).

[28] H. Yamaguchi, H. Uwe, T. Sakudo, and E. Sawaguchi, J. Phys. Soc. Jpn. 56, 589 (1987).

[29] I. Lubomirsky and O. Stafsudd, Rev. Sci. Instrum. 83, 051101 (2012).

[30] R. E. Cohen, Nature (London) 358, 136 (1992).

[31] G. Wang, L. Zhang, B. Yan, J. Luo, X. Su, and F. Yue, ChemCatChem 11, 1057 (2019).

[32] S. Jaiswar and K. D. Mandal, J. Phys. Chem. C 121, 19586 (2017).

[33] W. Li, R. Liang, A. Hu, Z. Huang, and Y. N. Zhou, RSC Adv. 4, 36959 (2014).

[34] G. Liu, H. G. Yang, X. Wang, L. Cheng, H. Lu, L. Wang, G. Q. Lu, and H. M. Cheng, J. Phys. Chem. C 113, 21784 (2009). 
[35] A. E. Bocquet, A. Fujimori, T. Mizokawa, T. Saitoh, H. Namatame, S. Suga, N. Kimizuka, Y. Takeda, and M. Takano, Phys. Rev. B 45, 1561 (1992).

[36] H. M. Nguyen, N. V. Dang, P.-Y. Chuang, T. D. Thanh, C.-W. Hu, T.-Y. Chen, V. D. Lam, C.-H. Lee, and L. V. Hong, Appl. Phys. Lett. 99, 202501 (2011).

[37] I. N. Apostolova, A. T. Apostolov, S. G. Bahoosh, and J. M. Wesselinowa, J. Appl. Phys. 113, 203904 (2013).

[38] S. Hashemizadeh, A. Biancoli, and D. Damjanovic, J. Appl. Phys. 119, 094105 (2016).

[39] S. I. Andronenko and S. K. Mishra, Appl. Magn. Reson. 46, 693 (2018).

[40] H. M. Rietveld, J. Appl. Crystallogr. 2, 65 (1969).

[41] H. M. Rietveld, E. N. Maslen, and C. J. B. Clews, Acta Crystallogr. Sect. B 26, 693 (1970).

[42] L. B. McCusker, R. B. Von Dreele, D. E. Cox, D. Louer, and P. Scardi, J. Appl. Crystallogr. 32, 36 (1999).

[43] L. W. Finger, D. E. Cox, and A. P. Jephcoat, J. Appl. Crystallogr. 27, 892 (1994).

[44] H. T. Langhammer, T. Müller, K. H. Felgner, and H. P. Abicht, J. Am. Ceram. Soc. 83, 605 (2000).
[45] S. G. Cao, Y. Li, H. H. Wu, J. Wang, B. Huang, and T. Y. Zhang, Nano Lett. 17, 5148 (2017).

[46] Z. Li, M. Yang, J. S. Park, S. H. Wei, J. J. Berry, and K. Zhu, Chem. Mater. 28, 284 (2016).

[47] S. Ray, D. D. Sarma, and R. Vijayaraghavan, Phys. Rev. B 73, 165105 (2006).

[48] E. R. Aluri and A. P. Grosvenor, J. Phys. Chem. 74, 830 (2013).

[49] O. Haas, U. F. Vogt, C. Soltmann, A. Braun, W. S. Yoon, X. Q. Yang, and T. Graule, Mater. Res. Bull. 44, 1397 (2009).

[50] D. C. Sinclair, J. M. S. Skakle, F. D. Morrison, R. I. Smith, and T. P. Beales, J. Mater. Chem. 9, 1327 (1999).

[51] D. Lu, Y. Zheng, and L. Yuan, Materials 12, 1525 (2019).

[52] J. H. Lee, N. C. Bristowe, J. H. Lee, S. H. Lee, P. D. Bristowe, A. K. Cheetham, and H. M. Jang, Chem. Mater. 28, 4259 (2016).

[53] A. Marthinsen, C. Faber, U. Aschauer, N. A. Spaldin, and S. M. Selbach, MRS Commun. 6, 182 (2016).

[54] J. Klarbring and S. I. Simak, Phys. Rev. B 97, 024108 (2018). 